\title{
OBTENÇÃO DE UBAIA DESIDRATADA PELO PROCESSO DE LIOFILIZAÇÃO
}

\section{OBTAINMENT OF DEHYDRATED UBAIA BY LYOPHILIZATION PROCESS}

\author{
Emanuel Neto Alves de Oliveira ${ }^{1}$; Dyego da Costa Santos ${ }^{2}$; Francinalva Cordeiro de Sousa ${ }^{3}$; Joabis \\ Nobre Martins ${ }^{4}$; Sônia Paula Alexandrino de Oliveira ${ }^{5}$ \\ ${ }^{1}$ Universidade Federal de Campina Grande - UFCG emanuelnetoliveira@ig.com.br \\ ${ }^{2}$ Universidade Federal de Campina Grande - UFCG dyego.csantos@ gmail.com \\ ${ }^{3}$ Faculdade de Tecnologia CENTEC - FATEC - E-mail: francis_nalva@yahoo.com.br \\ ${ }^{4}$ Faculdade de Tecnologia CENTEC - FATEC martins ta@ $@$ hotmail.com \\ ${ }^{5}$ Universidade Federal Rural de Pernambuco - UFRPE - soniadypaula@ hotmail.com
}

\begin{abstract}
Resumo
O presente estudo foi realizado com objetivo de obter ubaia desidratada pelo processo de liofilização e avaliar as alterações dos componentes nutricionais provocadas pelo processo. As amostras foram despolpadas no Laboratório de Processamento de Alimentos da Fatec-Cariri e a polpa liofilizada em liofilizador de bancada modelo L101. A fruta in natura e a polpa liofilizada foram submetidas às analises físico-químicas de acordo com as metodologias preconizadas em IAL (2004), Pearson (1971), AOAC (1975) e APHA (1992). A fruta in natura apresentou umidade inicial de 92,47\%, decrescendo para 14,25\%, após liofilizada. Houve concentração da vitamina $C$ de 43,72 mg/100g (fruta in natura) a 112,41 mg/100g (polpa liofilizada). Observou-se queda no $\mathrm{pH}$ $e$ aumento significativo nos teores de cinzas, acidez e tanino após a liofilização do fruto. As determinações de açúcares totais e sólidos solúveis totais apresentaram porcentagens de 4,39\% e $6,73{ }^{\circ}$ Brix para a fruta in natura e $10,6 \%$ e 7,75 ${ }^{\circ}$ Brix para a polpa liofilizada. Observou-se ainda aumento significativo nos teores de ferro, sódio e potássio e queda nos valores de fósforo. Concluise que o processo de liofilização não afetou significativamente as características originais da fruta in natura.
\end{abstract}

Palavras-chave: ubaia, secagem, componentes nutricionais.

\section{Introdução}

A procura pela diversificação de culturas proporcionou um aumento pelo interesse de cultivo e consumo de frutas exóticas. O aproveitamento de espécies frutíferas exóticas reflete na oferta de novas alternativas de frutas frescas para consumo e matéria-prima para agroindústria, constituindo uma preciosa fonte de alimentos (NASCIMENTO, 2008). A Eugenia pyriformis 
Camb. é uma espécie arbórea da família Mirtaceae, conhecida como ubaia, uvaia, uvalha (ANDRADE e FERREIRA, 2000).

Há certa divergência quanto ao nome científico desta fruta. Andrade e Ferreira (2000) e Lorenzi (2002) citam-na como Eugenia pyriformis Cambess., enquanto Andersen e Andersen (1988) e Scalon et al. (2004a) Scalon et al. (2004b), citam-na como Eugenia uvalha Cambess.

Os frutos, drupas globosas de cor amarela, são deiscentes, carnosos, contendo de uma a três sementes que apresentam tegumento de coloração castanha, cotilédones carnosos e justapostos. Após a extração, as sementes oxidam-se rapidamente, provocando um escurecimento nos cotilédones, que se inicia na periferia, indo em direção à parte central da semente. $O$ fruto é comestível, sendo muito apreciado na forma de sucos. Também é muito procurado por várias espécies de pássaros, o que o torna bastante recomendável para reflorestamentos heterogêneos destinados à recomposição da vegetação de áreas degradadas de preservação permanente (ANDRADE e FERREIRA, 2000).

A qualidade dos frutos é atribuída ao seu tamanho e forma e à cor da casca. Esses fatores, associados à composição físico-química da polpa, oferecem aos frutos e aos produtos deles obtidos a qualidade organoléptica e nutricional, responsáveis pela aceitação definitiva desses no mercado (SCALON et al., 2004a).

Atualmente tem-se empregado novas tecnologias que permitem processar a fruta na forma de pó, fazendo com que o fruto, que antes era consumido só em sua época de safra, hoje possa ser consumido em qualquer período do ano. O processo mais utilizado é a liofilização que faz com que a água contida no produto, passe do estado sólido (produto congelado) para o estado gasoso sem passar pelo estado líquido, ocorrendo desta forma, o processo de sublimação (IBARZ e BARBOSA-CÁNOVAS, 1999).

O presente estudo foi realizado com objetivo de obter ubaia desidratada pelo processo de liofilização e avaliar as alterações dos componentes nutricionais provocadas pelo processo.

\section{Material e Métodos}

\section{Obtenção das amostras}

A pesquisa foi realizada no segundo semestre de 2008. Os frutos foram obtidos no mercado varejista da cidade de Fortaleza-CE, e conduzidos à Faculdade de Tecnologia CENTEC, unidade de Juazeiro do Norte-CE. As amostras foram despolpadas no Laboratório de Processamento de Alimentos e liofilizadas em liofilizador de bancada modelo L101 do fabricante Liobras no Laboratório de Química de Alimentos da mesma Faculdade. 


\section{Análises físico-químicas}

As análises físico-químicas da fruta in natura e da polpa liofilizada foram realizadas em triplicata no Laboratório de Química de Alimentos, quanto aos parâmetros:

Umidade: Foi determinado pelo método de secagem das amostras até peso constante, em estufa a $105^{\circ} \mathrm{C}$, seguindo a metodologia descrita por Instituto Adolfo Lutz (2004).

Cinzas: As cinzas foram determinadas após completa carbonização em bico de bunsen e calcinadas em mufla a $550{ }^{\circ} \mathrm{C}$, até a obtenção de um resíduo com coloração branca acinzentada (IAL, 2004).

Vitamina $C$ : Foi empregado o método 2,6-diclorofenol indofenol em espectrofotômetro utilizando um comprimento de onda a $520 \mathrm{~nm}$, seguindo metodologia descrita por Pearson (1971).

Acidez total em ácido cítrico: A técnica utilizada foi a de titulometria volumétrica de neutralização com a solução de $\mathrm{NaOH} 0,1 \mathrm{~N}$ segundo normas analíticas do Instituto Adolfo Lutz (2004).

Determinação do $\mathrm{pH}$ : Utilizou-se a técnica potenciometrica através do pHmetro digital (IAL, 2004).

Tanino: Foi empregado o método de folin-denis em espectrofotômetro utilizando um comprimento de onda a $760 \mathrm{~nm}$, seguindo metodologia descrita por Pearson (1971).

Sólidos solúveis totais: Foi determinado utilizando um refratômetro de ABBE com escala de $0^{\circ}$ a $92^{\circ}$ Brix segundo Instituto Adolfo Lutz (2004).

Açúcares redutores: A técnica utilizada foi a de titulometria volumétrica com solução de Fehling segundo Instituto Adolfo Lutz (2004).

\section{Determinação dos minerais}

Fósforo: Foi empregado o método de vanadato-molibdato em espectrofotômetro utilizando um comprimento de onda a $470 \mathrm{~nm}$, seguindo metodologia descrita por Pearson (1971).

Ferro: A determinação de ferro foi realizada segundo método da orto-fenantrolina em espectrofotômetro utilizando um comprimento de onda a $510 \mathrm{~nm}$, de acordo com AOAC (1975).

Sódio e Potássio: Determinados segundo o método fotométrico de chamas descrito por APHA (1992).

\section{Resultados e Discussão}

Estão apresentados na Tabela 1 os resultados das análises físico-químicas de $\mathrm{pH}$, sólidos solúveis totais, teor de água, cinzas, acidez, açúcares redutores e vitamina $\mathrm{C}$ e tanino da fruta in natura e da polpa liofilizada. 
Tabela 01 - Análises físico-químicas da ubaia in natura e da polpa liofilizada

\begin{tabular}{ccc}
\hline Análises & \multicolumn{3}{c}{ Amostras } \\
\cline { 2 - 3 } & Fruta in natura & Polpa liofilizada \\
\hline $\mathrm{pH}$ & 2,70 & 2,01 \\
Sólidos solúveis totais ( ${ }^{\circ}$ Brix) & 6,73 & 7,75 \\
Umidade $(\%)$ & 92,47 & 14,25 \\
Cinzas (\%) & 0,39 & 5,73 \\
Acidez (\%) & 2,26 & 23,84 \\
Açúcares totais $(\%)$ & 4,39 & 10,60 \\
Tanino $(\mathrm{mg} / 100 \mathrm{~g})$ & 2,42 & 37,39 \\
Vitamina C (mg/100g) & 43,72 & 112,41 \\
\hline
\end{tabular}

A fruta in natura apresentou umidade inicial de 92,47\%, decrescendo para 14,25\% após liofilização. O teor de água da fruta in natura encontra-se superior ao citado por Carvalho (1988) que encontrou teor de $90,7 \%$. A determinação de umidade é uma das medidas mais importantes e utilizadas na análise de alimentos. O teor de umidade de um alimento está relacionado com sua estabilidade, qualidade e composição, e pode afetar o armazenamento, embalagens e processamento (CHAVES et al., 2004). Para Aldrigue et al. (2002), o conteúdo de umidade de um alimento é de grande importância por razões diversas, porém, sua determinação precisa é muito difícil, uma vez que a água ocorre nos alimentos de três diferentes maneiras: água ligada, água disponível e água livre.

O teor de vitamina C obtido na fruta in natura foi de 43,72 mg/100g. Donadio (1997) encontrou valores de vitamina $\mathrm{C}$ em frutos de ubaia variando de 33,00 a 39,52 mg/100g. A polpa de ubaia liofilizada revelou teor de vitamina $C$ de $112,41 \mathrm{mg} / 100 \mathrm{~g}$. A concentração de vitamina $\mathrm{C}$ era esperada, pois, ao se retirar água da furta in natura, há concentração dos demais constituintes, não sendo possível afirmar, no presente estudo, se houve perda de ácido ascórbico durante o processo. O ácido ascórbico é uma das vitaminas que mais pode ser alterada no processamento dos alimentos, contribuindo para isso o fato de ser hidrossolúvel, a ação do calor, da luz, do oxigênio, de álcalis, da oxidase do ácido ascórbico, bem como traços de cobre e ferro. Sua conservação é favorecida em meio ácido (TAVARES et al., 2003).

Observa-se na Tabela 1 que houve queda no $\mathrm{pH}$ de 2,7 a 2,01 e aumento significativo nos teores de cinzas, acidez e tanino após a liofilização do fruto (Figura 1).

A determinação de acidez pode fornecer um dado valioso na apreciação do estado de conservação de um produto alimentício. Um processo de decomposição seja por hidrólise, oxidação ou fermentação, altera quase sempre a concentração dos íons de hidrogênio (IAL, 2004). 
FIGURA 01 - Análises físico-químicas da fruta in natura e da polpa liofilizada

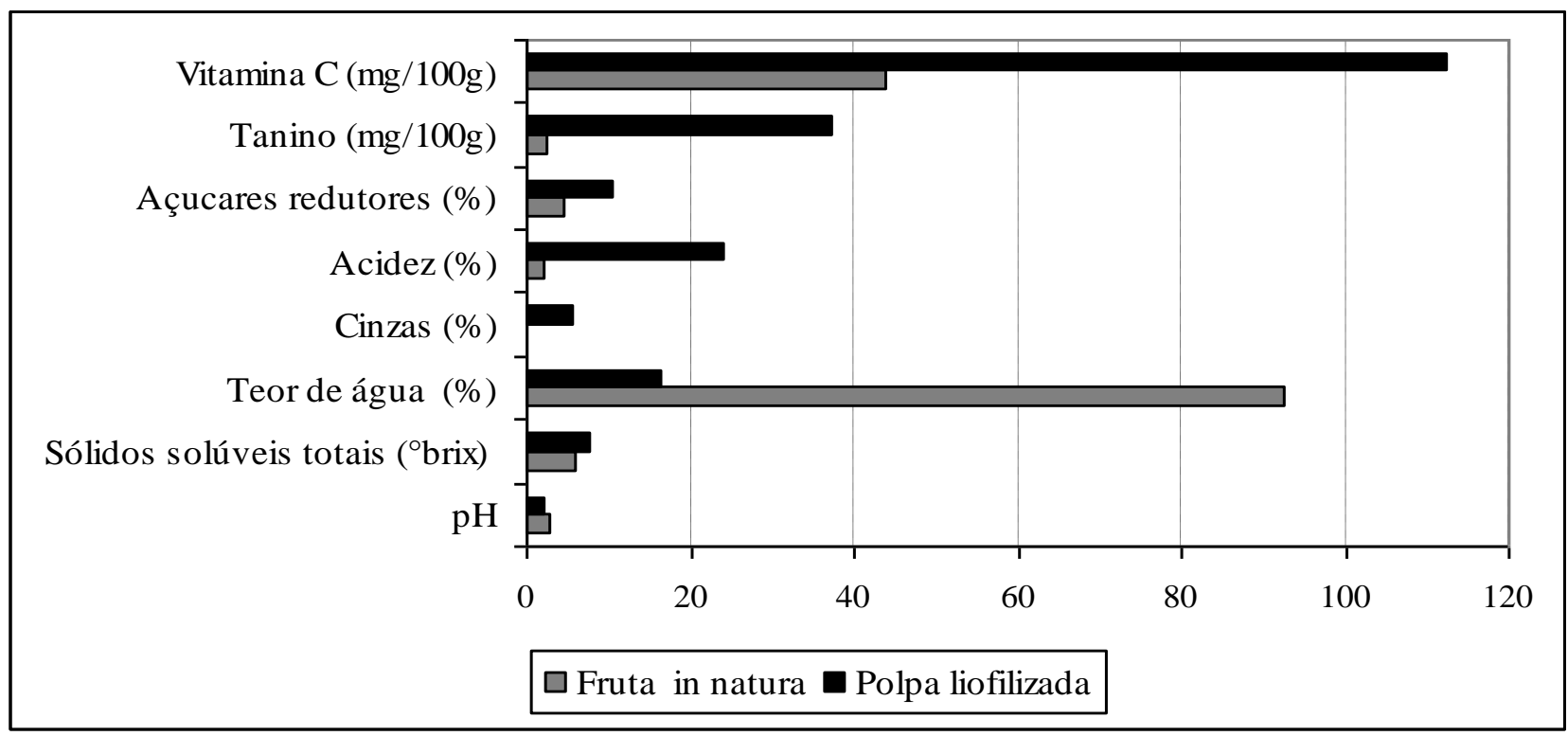

A determinação de acurares totais e sólidos solúveis totais apresentaram porcentagens de $4,39 \%$ e $6,73{ }^{\circ}$ Brix para a fruta in natura e $10,60 \%$ e $7,75{ }^{\circ}$ Brix para a polpa liofilizada, respectivamente. Os valores encontrados para fruta in natura são semelhantes aos citados por Donadio (1997) para açúcares totais e Scalon et al. (2004a) para sólidos solúveis totais.

Observam-se, na Tabela 2, os resultados das analises de minerais: ferro, fósforo, sódio e potássio para a fruta in natura e polpa liofilizada.

TABELA 02 - Análises de minerais da ubaia in natura e da polpa liofilizada

\begin{tabular}{ccc}
\hline & \multicolumn{2}{c}{ Amostras } \\
\cline { 2 - 3 } Análises (mg/100g) & Fruta in natura & Polpa liofilizada \\
\hline Ferro & 7,16 & 12,71 \\
Fósforo & 297,02 & 111,05 \\
Sódio & 84,05 & 376,74 \\
Potássio & 643,01 & 1523,09 \\
\hline
\end{tabular}

Para o mineral ferro houve concentração de 7,16 mg/100g (ubaia in natura) para 12,71 $\mathrm{mg} / 100 \mathrm{~g}$ (ubaia liofilizada).

Observa-se ainda aumento significativo nos teores de sódio e potássio e queda nos valores de fósforo (Figura 2). 
FIGURA 02 - Análises de minerais da ubaia in natura e da ubaia liofilizada.

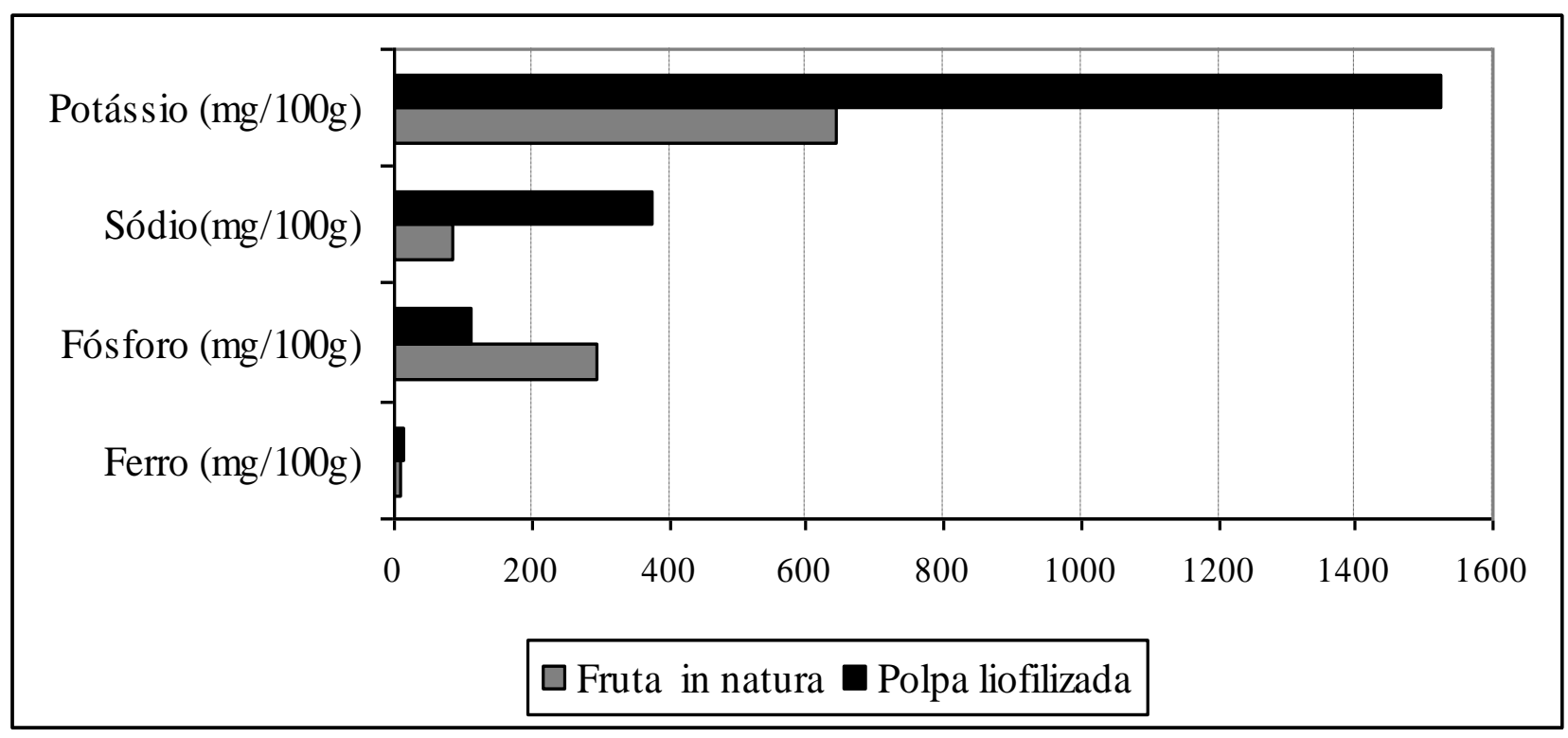

\title{
4. Conclusões
}

Diante dos resultados, conclui-se que o processo de secagem de alimentos por liofilização preserva em grande parte as características originais do produto in natura, destacando-se o teor de vitamina $\mathrm{C}$, concentrada após o processo.

\begin{abstract}
This study was carried out aiming to obtain ubaia dehydrated by lyophilization process and evaluate the changes of the nutritional components caused by the process. The pulp samples were processed in the Laboratory of Food Processing of Fatec-Cariri and lyophilized in lyophilizer bench model L101. The fruit in nature and lyophilized pulp were subjected to physical and chemical analysis in accordance with the methodologies outlined in IAL (2004), Pearson (1971), AOAC (1975) and APHA (1992). The fruit in nature had an initial moisture content of $92.47 \%$, falling to $14.25 \%$ after lyophilized. There was a concentration of vitamin $C$ of $43.72 \mathrm{mg} / 100 \mathrm{~g}$ (fruit in nature) to $112.41 \mathrm{mg} / 100 \mathrm{~g}$ (pulp lyophilized). There was a reduction in $\mathrm{pH}$ and significant increase in ash content, acidity and tannin after lyophilization of the fruit. The determination of total sugars and soluble solids total presented percentages of $4.39 \%$ and $6.73{ }^{\circ}$ Brix for the fruit in nature and $10.6 \%$ and $7.75^{\circ}$ Brix for the pulp lyophilized. There was also a significant increase in levels of iron, sodium and potassium and reduced values of phosphorus. We conclude that freeze-drying did not affect the original characteristics of fruit in nature.
\end{abstract}

Key-words: ubaia, drying, nutritional components.

\section{Referências}

ALDRIGUE, M. L.; MADRUGA, M. S.; FIOREZE, R.; LIMA, A. W. O.; SOUSA, C. P. Aspecto da ciência e tecnologia de alimentos. João Pessoa: Ed. UFPB, 2002. 
APHA (American Public Health Association). Standard Methods For The Examination Of Dairy Products. 16. ed. Washington: APHA, 1992.

AOAC (Association of Official Agricultural Chemists). Official Methods Of Analysis Of AOAC International. 12. ed. Washington: AOAC International. 1975. 1094p.

ANDERSEN, O.; ANDERSEN, V. U. As frutas silvestres brasileiras: uvaia. Rio de Janeiro: Globo, p. 198-200, 1988.

ANDRADE, R. N. B. de; FERREIRA, A. G. Germinação e armazenamento de sementes de uvaia (Eugenia pyriformis Camb.) - Myrtaceae. Revista Brasileira de Sementes, v. 22, n. 2, p. 118-125, 2000.

CARVALHO, P. R. N. Análises de vitaminas em alimentos: manual técnico. Campinas: Instituto de Tecnologia de alimentos, 1988.

CHAVES, M. C. V.; GOUVEIA, J. P. G.; ALMEIDA, F. A. C.; LEITE, J. C. A.; SILVA, F. L. H. Caracterização físico-química do suco da acerola. Revista de Biologia e Ciências da Terra, v. 4, n. 2, 2004.

DONADIO, L.C. Study of some Brazilian Myrtaceae in Jaboticabal - SP. Acta Horticulturae, v. 452, p. 181-183, 1997.

IBARZ, A.; BARBOSA-CÁNOVAS, G. V. Operaciones unitárias de la ingenieria de alimentos. Lancaster: Technomic Publishing. 1999.

INSTITUTO ADOLFO LUTZ. Normas Analíticas do Instituto Adolfo Lutz: Métodos Químicos e Físicos Para Análise de Alimentos. 3 ed. São Paulo: IAL, 2004.

LORENZI, H. Árvores brasileiras: manual de identificação e cultivo de plantas arbóreas do Brasil. 4 ed, v. 14. Nova Odessa: Instituto Plantarum de Estudos da Flora, p. 277, 2002.

NASCIMENTO, V.E. Caracterização de plantas de mamey. 53f. Dissertação (Mestrado em Agronomia). Universidade Estadual Paulista "Julio de Mesquita Filho". Faculdade de Ciências Agrárias e Veterinárias, Jaboticabal, 2008 .

PEARSON, D. The chemical analysis of foods. 6. ed. New York: Hemical Publ., 1971. 604p.

SCALON, S. P. Q.; DELL'OLIO, P; FORNASIERI, J. L. Temperatura e embalagens na conservação pós-colheita de uvaia Eugenia uvalha Cambess.- Mirtaceae. Ciência Rural, v. 34, n. 6, p. 1965-1968, 2004a. DOI:10.1590/S010384782004000600048 .

SCALON, S de P. Q.; SCALON FILHO, H; RIGONI, M. R. Armazenamento e germinação de sementes de uvaia Eugenia uvalha Cambess. Ciência e Agrotecnologia, v. 28, n. 6, p. 1228-1234, 2004b. DOI: 10.1590/S141370542004000600002 .

TAVARES, J. T. Q.; SANTOS, C. M. G.; TEIXEIRA, L. J.; SANTANA, R. S.; PORTUGAL, A. M. Estabilidade do ácido ascórbico em polpa de acerola submetida a diferentes tratamentos. Magistra on line. v. 15, n. $2,2003$.

\section{Dados dos autores:}

Nome completo: Emanuel Neto Alves de Oliveira

Filiação institucional: UFCG

Departamento: Engenharia Agrícola

Função ou cargo ocupado: Mestrando em Engenharia Agrícola

Titulação: Especialista em Ensino de Química

Endereço: Av. Aprígio Veloso, 882 - Bodocongó - Caixa Postal 10.078 - CEP 58109-970 Campina Grande, PB - Brasil

Telefones para contato: (83) 33101055 e-mail: emanuelnetoliveira@ig.com.br 
Nome completo: Dyego da Costa Santos

Filiação institucional: UFCG

Departamento: Engenharia Agrícola

Função ou cargo ocupado: Mestrando em Engenharia Agrícola

Titulação: Tecnólogo em Alimentos

e-mail: dyego.csantos@gmail.com

Nome completo: Francinalva Cordeiro de Sousa

Filiação institucional: FATEC - Cariri-CE

Departamento: Tecnologia de Alimentos

Função ou cargo ocupado: Laboratorista

Titulação: Especialista em Ensino de Química

e-mail: francis_nalva@yahoo.com.br

Nome completo: Joabis Nobre Martins

Filiação institucional: FATEC - Limoeiro do Norte-CE

Departamento: Tecnologia de Alimentos

Titulação: Tecnólogo em Alimentos

e-mail: $\underline{\text { martins_ta@hotmail.com }}$

Nome completo: Sonia Paula Alexandrino de Oliveira

Filiação institucional: UFRPE

Departamento: Tecnologia de Alimentos

Função ou cargo ocupado: Mestranda em Ciência e Tecnologia de Alimentos

Titulação: Tecnóloga em Alimentos

e-mail: soniadypaula@hotmail.com 